\title{
Secondary Postpartum Hemorrhage due to Spontaneous Uterine Artery Rupture after Normal Vaginal Delivery Managed by Selective Arterial Embolization
}

\author{
Mili Thakur, MD ${ }^{1,2}$ Henry O. Adekola, MD ${ }^{3}$ Radwan Asaad, MD ${ }^{1} \quad$ Bernard Gonik, MD ${ }^{1}$ \\ ${ }^{1}$ Department of Obstetrics and Gynecology, Wayne State University \\ School of Medicine/Detroit Medical Center, Detroit, Michigan \\ 2 Division of Genetic and Metabolic Disorders, Department of \\ Pediatrics and Center for Molecular Medicine and Genetics, Wayne \\ State University/Detroit Medical Center, Detroit, Michigan \\ ${ }^{3}$ Division of Maternal Fetal Medicine, Department of Obstetrics and \\ Gynecology, Hurley Medical Center, Flint, Michigan
Address for correspondence Mili Thakur, MD, Division of Reproductive Endocrinology and Infertility, Department of Obstetrics and Gynecology, Wayne State University, Detroit, MI, 48201 (e-mail: mthakur@med.wayne.edu). \\ Am J Perinatol Rep 2016;6:e442-e444.
}

\author{
Abstract \\ Keywords \\ - secondary \\ postpartum \\ hemorrhage \\ - spontaneous uterine \\ artery rupture \\ - uterine artery \\ embolization \\ - intraperitoneal \\ bleeding \\ - hemorrhagic shock
}

Secondary postpartum hemorrhage due to an intraperitoneal bleed following a vaginal delivery is extremely rare. We present a case of spontaneous rupture of the uterine artery following a normal vaginal delivery with a delayed presentation, which resulted in significant morbidity. This case discusses the presentation and management of this rare obstetrical emergency. The report also discusses the role of selective arterial embolization in management of secondary postpartum hemorrhage.
Secondary postpartum hemorrhage (PPH) is hemorrhage commencing more than 24 hours following delivery until 6 weeks postpartum. It is an unusual complication following a vaginal delivery with a reported incidence of 0.5 to $1.3 \%$, but is associated with serious maternal morbidity and mortality. ${ }^{1}$ Intraperitoneal hemorrhage, following a vaginal delivery, is a very rare and unanticipated cause of secondary postpartum hemorrhage. Bleeding from utero-ovarian vessels, ${ }^{2-4}$ ruptured aneurysm of the splenic artery, ${ }^{5}$ and the Santorini plexus in the space of Retzius ${ }^{6}$ have been reported. Only six cases of spontaneous rupture of uterine artery in puerperium after an uncomplicated vaginal delivery have been reported. ${ }^{3,4}$

received

October 25, 2016 accepted after revision November 12, 2016
DOI http://dx.doi.org/ 10.1055/s-0036-1597623. ISSN 2157-6998.

\section{Case}

A 39-year-old multiparous woman underwent a spontaneous vaginal delivery following induction of labor at 38 weeks' gestation for chronic hypertension with superimposed preeclampsia. The delivery was atraumatic without any perineal or cervical lacerations, but complicated by uterine atony and primary postpartum hemorrhage with $800 \mathrm{~mL}$ estimated blood loss. Management included bimanual massage, oxytocin, and carboprost administration. Her postpartum hemoglobin was $8.6 \mathrm{~g} / \mathrm{dL}$. The patient remained hemodynamically stable and was discharged home on postpartum day 2 . She presented to the emergency department on postpartum day 3
Copyright $\odot 2016$ by Thieme Medical
Publishers, Inc., 333 Seventh Avenue,
New York, NY 10001, USA.
Tel: $+1(212)$ 584-4662. 
with acute abdominal pain and clinical evidence of hypovolemic shock. There was minimal vaginal lochia and the uterus was firm to palpation. Focused assessment with sonography for trauma (FAST) demonstrated hemoperitoneum. Immediate exploratory laparotomy was performed. A large retroperitoneal hematoma was identified arising from the pelvis. Because of the severity and degree of the hematoma infiltration, the source of bleeding could not be identified with limited dissection. Abdominal packing was performed. Negative-pressure wound therapy (external wound vacuum-assisted closure [VAC]) was placed. The massive transfusion protocol was in place in an attempt to stabilize the patient. A transfemoral arteriogram was performed, which demonstrated a large expanding area of hemorrhage arising from the left

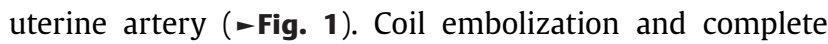
occlusion of the anterior division of left internal iliac artery were achieved (-Fig. 2 ). The patient remained in the intensive care unit for 5 weeks. During this time, she was taken back to the operating room six times for abdominal washout and VAC replacement. She had multiple postoperative complications, including an episode of cardiorespiratory arrest, respiratory failure requiring tracheostomy, bilateral lower extremity and right arm deep venous thrombosis, pulmonary embolism, acalculous cholecystitis, and bacterial sepsis. In total she received 34 units of packed red blood cells, 14 units of fresh frozen plasma, and 20 units of platelet concentrates. After interventions for the aforementioned morbidities, the patient's condition stabilized and she was eventually discharged to continue her recovery as an outpatient.

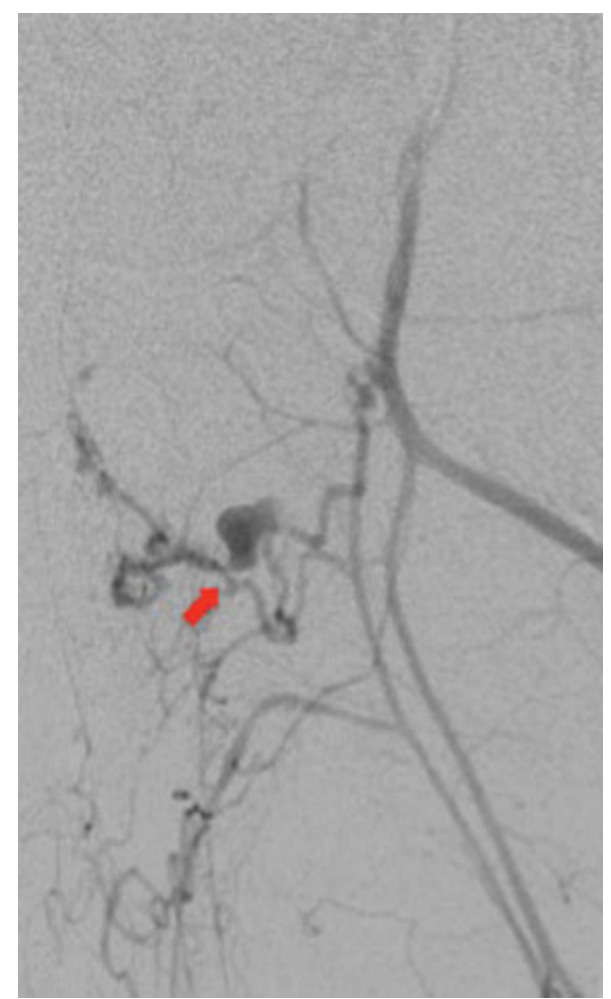

Fig. 1 Transfemoral arteriogram demonstrating a large expanding area of hemorrhage arising from the left uterine artery.

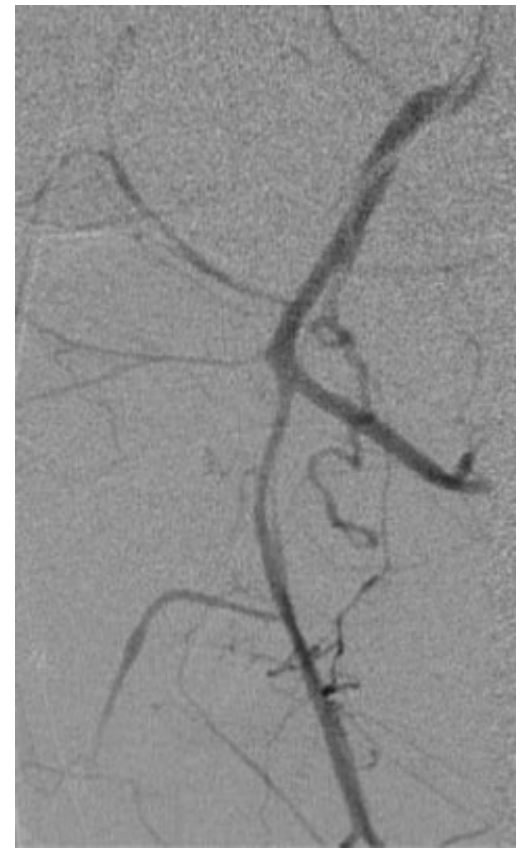

Fig. 2 Achieving coil embolization and complete occlusion of anterior division of left internal iliac artery.

\section{Discussion}

This case of secondary PPH presents a rare obstetrical emergency. It demonstrates the potential catastrophe associated with delayed presentation of spontaneous rupture of the uterine artery in the immediate puerperium. Hypovolemic shock without significant external bleeding, abdominal pain, palpable abdominal mass, or peritoneal signs are the usual presenting features. ${ }^{3,4}$ Initial management includes aggressive fluid resuscitation and/or transfusion of blood and blood products. An immediate laparotomy is warranted when intraperitoneal hemorrhage is identified. Hematoma evacuation and ligation of the bleeding vessel should be attempted. However, in some cases such as ours, the source of bleeding cannot be identified. Depending on the intraoperative findings, treatment by the use of surgical compression sutures, hysterectomy, and pelvic arterial ligation are appropriate interventions. ${ }^{7}$ In some cases, despite these measures, the bleeding continues and abdominopelvic packing is used to attenuate hemorrhage in order to stabilize the patient while a definitive approach is attempted.

Embolization of the bleeding artery is a reasonable consideration when surgical intervention cannot guarantee control of the bleeding site. Utilizing access to the concerned artery through catheters and guidewires and extravasation of contrast material as studied by digital subtraction angiography, an interventional radiologist locates the position of the correct artery or vein supplying the site of the bleeding. Embolization of the selective artery is then performed using a variety of materials including coils, foam, microspheres, and particles. The prerequisites for selective embolization are that the patient should be hemodynamically stable and that an experienced radiologist with the required infrastructure is available. 
Selective arterial embolization has been associated with a high clinical effectiveness rate and a low complication rate in appropriate patients. In a study of 66 women undergoing uterine artery embolization for PPH, the overall clinical success rate was $95 \%$ and the overall complication rate was $4.5 \%{ }^{8}$

Hemodynamic changes associated with pregnancy, congenital arterial venous malformations, inflammatory processes, and arterial degeneration have been reported as possible causes of this catastrophic complication. ${ }^{9}$ As a biopsy of the affected artery was not obtained for microscopic examination, the etiology of the uterine artery rupture in this case remains unclear. Similar to this case, the left uterine artery is more commonly involved in the rupture. ${ }^{3,4}$ Factors associated with the increased frequency of rupture of the left uterine artery include dextrorotation of the uterus and left occipitotransverse/left occipitoanterior position the fetal head. ${ }^{4}$ An extensive search of literature did not reveal any association of bimanual uterine massage with spontaneous rupture of pelvic vessels.

Although multiple adverse pregnancy outcomes have been reported after elective uterine artery embolization for management of fibroids including decreased ovarian reserve, increased risk of miscarriage, abnormal placentation, fetal malpresentation, increased cesarean delivery and $\mathrm{PPH},{ }^{10}$ the data on pregnancies after uterine artery embolization after PPH are limited. However, most patients resumed menstruation and some have successfully carried subsequent pregnancies. $^{11}$

This case is the second case of spontaneous uterine artery rupture reported with delayed presentation after vaginal delivery $^{3}$; however, it is the first reported case in which selective artery embolization was successfully used for management. In conclusion, this case highlights a rare presentation of secondary postpartum hemorrhage due to spontaneous rupture of the uterine artery. The availability of a multidisciplinary team, including the use of selective uterine artery embolization, was vital in averting maternal mortality despite significant morbidity.

\section{Conflict of Interest}

The authors report no conflict of interest.

\section{Established Facts}

- Secondary postpartum hemorrhage due to intraperitoneal bleed is rare.
- Management includes aggressive resuscitation, uterotonics, surgical intervention, and selective arterial embolization.

\section{Novel Insights}

Delayed intraperitoneal hemorrhage following a vaginal delivery may take the emergency room physician and obstetrician by surprise. Hypovolemic shock without external bleeding should raise the suspicion for intraperitoneal hemorrhage. Multiple surgical techniques may be required to stop bleeding. There is a role of selective arterial embolization in managing this rare cause of secondary postpartum hemorrhage.

\section{References}

1 Hoveyda F, MacKenzie IZ. Secondary postpartum haemorrhage: incidence, morbidity and current management. BJOG 2001; 108(9):927-930

2 Banaś T, Boryczko M, Durzyńska-Urbaniec J. [Intraperitoneal hemorrhage due to the rupture of right ovarian artery in the second day of puerperium] in Polish]. Ginekol Pol 2004;75(9): 729-732

3 Becker R, Kowalsky BL, Hatzmann W. [Rupture of the uterine artery after spontaneous delivery with unusual course in childbed] [in German]. Z Geburtshilfe Neonatol 2002;206(3):107-113

4 Duhan N, Sangwan N, Rajotia N, Kadian YS, Singla SL. Spontaneous uterine artery rupture at delivery. J Obstet Gynaecol India 2013; 63(1):72-73

5 Asokan S, Chew EK, Ng KY, Thanaletchimy N, Asmiati A, Kong NM. Post partum splenic artery aneurysm rupture. J Obstet Gynaecol Res 2000;26(3):199-201

6 Fieni S, Berretta R, Merisio C, Melpignano M, Gramellini D. Retzius' space haematoma after spontaneous delivery: a case report. . Acta Biomed 2005;76(3):175-177

7 Morel O, Malartic C, Muhlstein J, et al. Pelvic arterial ligations for severe post-partum hemorrhage. Indications and techniques. J Visc Surg 2011;148(2):e95-e102

8 Ganguli S, Stecker MS, Pyne D, Baum RA, Fan CM. Uterine artery embolization in the treatment of postpartum uterine hemorrhage. J Vasc Interv Radiol 2011;22(2):169-176

9 Ginsburg KA, Valdes C, Schnider G. Spontaneous utero-ovarian vessel rupture during pregnancy: three case reports and a review of the literature. Obstet Gynecol 1987;69(3 Pt 2):474-476

10 Ravina JH, Vigneron NC, Aymard A, Le Dref O, Merland JJ. Pregnancy after embolization of uterine myoma: report of 12 cases. Fertil Steril 2000;73(6):1241-1243

11 Deux JF, Bazot M, Le Blanche AF, et al. Is selective embolization of uterine arteries a safe alternative to hysterectomy in patients with postpartum hemorrhage? AJR Am J Roentgenol 2001;177(1): 145-149 